(NASA-CR-199628) A C-BAND

BACKSCATTER MODEL FOR LAKE ICE IN

ALASKA (National Space Development Agency) $4 \mathrm{p}$

N96-14295

Unclas

$63 / 45 \quad 0076337$ 


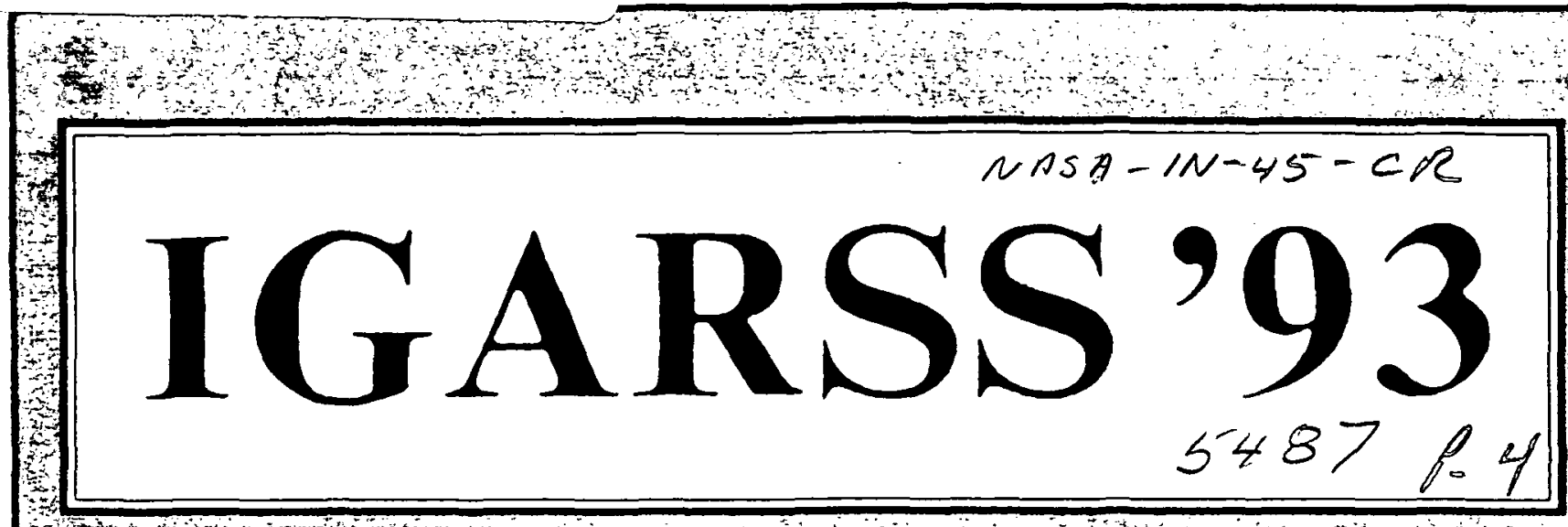



\section{Better Understanding of \\ 2. Earth Environment}
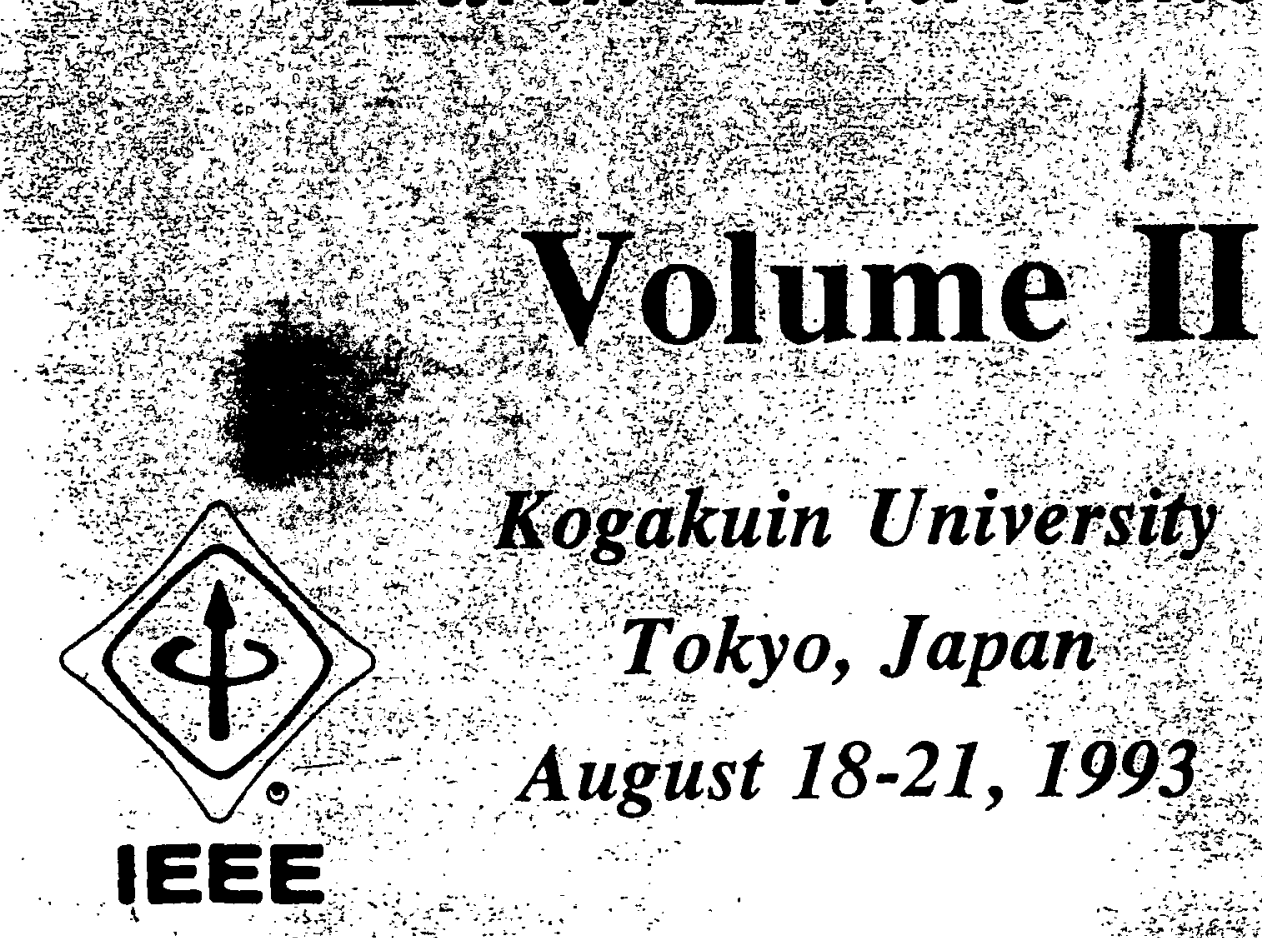

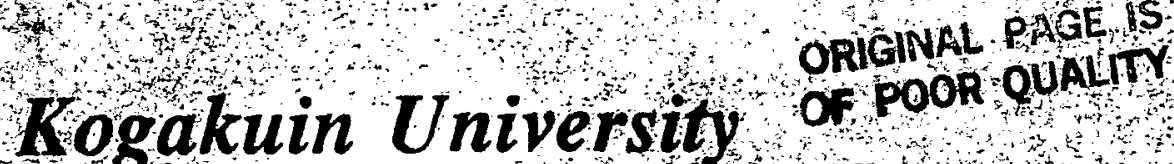
Tokyo, Japan August 18-21, 1993

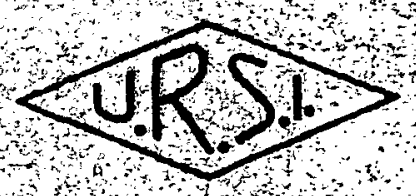

IEEE Catalog Number: 93CH32946

Library of Congress Number: $93-77594$ 


\title{
A C-BAND BACKSCATTER MODEL FOR LAKE ICE IN ALASKA
}

\author{
H. Wakabayashi 1, W. F. Weeks 2 and M. O. Jeffries 2 \\ 1: Earth Observation Center/NASDA, Saitama 350-03, Japan \\ 2: Geophysical Institute, University of Alaska Fairbanks, Fairbanks, AK 99775-0800, U.S.A.
}

\section{ABSTRACT}

ERS-1 SAR imagery of lake ice growing on shallow tundra lakes in northern Alaska shows interesting radar backscatter variations. Based on the analysis of ice cores from these lakes, a multi-layer backscatter model comprised of the following elcunents has heen develoned: I) specular air-ice. ice-water and ice-frozen soil boundaries; 2 ) an ice layer of variable thickness; 3) ice sub-layers with air inclusions of variable density, size and shape. including spheres, prolate spheroids, and cylinders of finite length. Preliminary model results confirm that backscatter is a sensitive function of the presence of a specular ice-water interface, with a roughly 40 -times greater reflectivity than from an ice-frozen soil interface. The inodel has also heen tested using bubble data derived from ice cores in April. 1992. The modelled backscatter is compared with backscatter derived from ERS-1 SAR images obtained at the same time as the fieldwork.

Keyword: backscatter model, radar backscatter, lake ice, tundra lakes

\section{INTRODUCTION}

The North Slope of Alaska is a large expanse of low-lying tundra with many shallow lakes covering more than $40 \%$ of the surface area. During the 1970s, in late winter, SLAR images of these lakes showed interesting variations of backscatter intensity, with areas of low backscatter at lake margins believed to represent ice that was frozen to the lake bed, while areas of high backscatter were considered to represent floating ice that contained tubular bubbles which acted as forward scatterers [Seliman et al..1975; Elachi et al.,1976: Weeks et al.,1977,1978.19811.

From September 1991 to April 1992, backscatter intensity variations from shallow lundra lakes near Barrow, NW Alaska, were studied using C-band SAR data from the ESA Remote-Sensing Satellite-1 (ERS-1). The SAR data were processed at the Alaska SAR Facility (ASF) and backscattering coefficients were derived for a number of lakes. Backscatter intensity varied from $-19 \mathrm{~dB}$ to $-6 \mathrm{~dB}$. Field measurements in April 1992 confirmed that the highest values were associated with floating ice containing tubular bubbles. while the lowest backscatter values were associated with ice frozen to the lake bed. but which also contained tubular bubbles (Jeffries et al., 1993).

This paper reports on the development of the first numerical model of $\mathrm{C}$ hand backscatter from such ice covered, shallow tundra lakes. Backscatter is simulated for grounded and floating ice of variable thickness and stratigraphic characteristics. Also. using ice physical characteristics observed and measured in late April as inputs to the model, simulated backscatter is compared with backscatter derived from ERS-1 SAR images obtained at a similar time.

\section{MODELING}

\subsection{Multi-layer consideration}

In April 1992, field measurements were made at 11 locations near Barrow, NW Alaska. Ice cores were also obtained from 10 sites and taken to the laboratory for detailed ice core analysis, which indicated the presence of three ice types: 1) granular ice containing roughly spherical bubbles, with radii much smaller than a wavelength ( $<5.7 \mathrm{~cm}) ; 2$ ) clear bubble-free ice; and. 3) ice containing lubular bubbles resembling thin cylinders. The latter, oriented vertical to the ice surface. also had radii much smaller than a wavelength. but with lengths ranging from $1.5 \mathrm{~cm}$ and $9.1 \mathrm{~cm}$. The number of tubular bubbles per square meter, i.e. bubble density or ice porosity, was quite variable |Jeffries et al., 1993|.

The typical stratigraphy of the lake ice is shown in Fig. 1. The clear ice layer and one or more tubular bubble layers were found at all sites. while the granular ice layers were found at 6 of the 10 sites. Each layer had a different thickness, bubble density and bubble size, especially in the tubular bubble layers where the bubble density and bubble size varied considerably with depth. The snowcover was of variable depth $(0-80 \mathrm{~mm})$ and dry.

Considering the features described above, our basic lake ice model is comprised of the following elements:

(1) ihree main layers (either air-ice-water or air-ice-frozen snil);

(2) sub-layers in the ice layer with each sub-layer having a different bubble density and bubble size (a bubble density of zero corresponds to clear ice):

(3) the boundaries between the main layers are considered to be specular:

(4) reflections occiur at the main layer boundaries and at the air bubbles; and,

(5) dielectric parameters of the ice layers change with the bulk ice density. i.e. with the density of bubbles present in the ice.

Because the surface roughness on these lakes is generally considered to be small compared to other sources of scattering, surface scattering is neglected. The dry snowcover is also neglected. For dry snow, the dielectric mismatch at the air-snow boundary is very small and the reflection at the air-snow boundary can be ignored. In addition. since the sriowcover was thin and snow particles very small, the contribution of the snowcover to the backscattering coefficient may be neglected at both L- and C-band frequencies |Ulaby et al.. 1986). It should be noted that reflections are added incoherently in the model, because the length of each path is widely distributed. Calculations are made on the basis of power.

The scattering coefficient of an extended target in a given direction is the ratio of the tolal scattered power from an equivalent isotropic scatterer which generates the same scattered power density in the direction to the total power of the illumination area. If the incident and scattering intensities are given, the backscattering coefficient would be as follows:

$$
\sigma_{\infty}^{0}=4 \pi \cos \theta_{0} \cdot \frac{l_{p}^{s}}{l_{q}^{i}}
$$

where $\theta_{0}$ is the incident and scattered angle, $I_{p}^{s}$ is the scattered p-polarized intensity, and $l_{q}^{i}$ is the incident q-polarized intensity. When reflections by bubbles in the ice occur, the scattered power is propagated to the backscaltered direction, as shown schematically in Fig. 2 . In the general case, the bubble density and bubble size vary according to the depth of ice. Thus, it is assumed that the ice is composed of $n$-layers and each layer has a different bubble density and bubble size. The downward and the upward intensities $(\bar{D}, \bar{U})$ at boundaries of layer $i$ are defined as shown in Fig. 3. The relation of these intensities is given by

$$
\left[\begin{array}{c}
\tilde{D}_{i} \\
\tilde{U}_{i-1}
\end{array}\right]=\left[\begin{array}{cc}
M_{a} & M_{b} \\
M_{c} & M_{d}
\end{array}\right]\left[\begin{array}{c}
\tilde{D}_{i-1} \\
\tilde{U}_{i}
\end{array}\right]
$$

where

$\bar{D}_{i}=\left[\begin{array}{ll}D_{i}^{*} & D_{i}^{-}\end{array}\right]^{\prime}, \bar{D}_{i-1}=\left[\begin{array}{ll}D_{i-1}^{*} & D_{i-1}^{-}\end{array}\right]^{\prime}, \bar{U}_{i}=\left[\begin{array}{ll}U_{i}^{*} & U_{i}^{-}\end{array}\right]^{\gamma}, \bar{U}_{i-1}=\left[\begin{array}{ll}U_{i-1}^{*} & U_{i-1}^{-}\end{array}\right]^{\prime}$. Here $t$ indicates a transposed matrix, and $M_{d}, M_{b}, M_{c}$ and $M_{d}$ are $2 \times 2$ matrices, of which the elements are given by the calculation for the first order scattering. For convenience in the multi-layer treatment, the following matrix for each layer is calculated.

$$
\left[\begin{array}{l}
\tilde{D}_{i-1} \\
\tilde{U}_{i-1}
\end{array}\right]=\left[\begin{array}{cc}
M_{a}^{-1} & -M_{a}^{-1} M_{b} \\
M_{r} M_{a}^{-1} & M_{d}-M_{c} M_{a}^{-1} M_{b}
\end{array}\right]\left[\begin{array}{l}
\tilde{D}_{i} \\
\tilde{U}_{i}
\end{array}\right]
$$

In order to obtain the relation between the incident and scattering intensities at the air-ice boundary, this matrix for each layer is derived and multiplied. and finally, the matrix for entire ice layer $K$ derived according to.

$$
\left[\begin{array}{l}
\bar{D}_{n} \\
\dot{U}_{n}
\end{array}\right]=K\left[\begin{array}{l}
\tilde{D}_{n} \\
\tilde{U}_{n}
\end{array}\right]=\left[k_{l m}\right]\left[\begin{array}{l}
\tilde{D}_{n} \\
\bar{U}_{n}
\end{array}\right]
$$


where $k_{t m}$ is the element of the matrix in row $l$ and column $\mathrm{m}$. Because there is no upward intensity from the bottom layer (water or frozen soil) and no left downward intensity in the upper layer (air), the following conditions must be satisfied.

$$
D_{0}^{*}=I^{i} \cdot D_{0}^{-}=U_{n}^{*}=U_{n}^{-}=0
$$

Using the above condition. the ratio of backscattering intensity to the incident intensity is given by.

$$
\frac{I^{*}}{r^{i}}=\left(1-\Gamma_{0}\right) \cdot \frac{U_{0}^{*}}{D_{0}^{+}} \cdot \frac{k_{41}-\frac{k_{42} k_{21}}{k_{22}}}{k_{11}-\frac{k_{12} k_{21}}{k_{22}}}
$$

Substituting Eq.(5) to Eq.(1), we obtain the backscattering coefficient as follows.

$$
\sigma^{0}=4 \pi \cos \theta_{0} \cdot\left(1-\Gamma_{0}\right) \cdot \frac{k_{41}-\frac{k_{42} k_{21}}{k_{22}}}{k_{11}-\frac{k_{12} k_{21}}{k_{22}}}
$$

\subsection{Bubble scattering}

In order to obtain each element in the $4 \times 4$ matrix of Eq.(2), the reflectivity of each boundary, the volume extinction coefficient, and the bistatic cross section for bubbles are required. The power reflectivity can be calculated by applying Fresnel's law using the average permittivity of the ice layer, which is considered to be a "two phase mixture" composed of pure ice and air bubbles [deLoor, 1968). The volume extinction coefficient is the sum of the volume absoiption coefficient and volume scattering coefficient.

The air bubbles in the granular ice layer are modelled as spheres with a size much smaller than a wavelength. In the tubular bubble ice layer, the air bubbles are modelled as either prolate spheroids or cylinders of finite length according to the length of bubbles. Because the radii of the bubbles are much smaller than a wavelength for all cases. low frequency approximation is applied to obtain the bistatic cross section. The scattering formulation used in this model is that given by Ruck et al. (1970).

The geometry of the problem is shown in Fig. 4. The bistatic VV cross sections for spheres $\sigma^{\text {ph }}\left(\psi_{i}, \psi_{,}, \phi\right)$, for prolate spheroids $\sigma^{2 / p}\left(\psi_{i}, \psi_{s}, \phi\right)$, and for cylinders of finite length $\sigma^{x}\left(\psi_{i}, \psi_{s}, \phi\right)$ are given as follows;

$\sigma^{2+(}\left(\psi_{1}, \psi_{,} \phi\right)=4 \pi a^{2}(k a)^{4} \mid \frac{1-\left.\varepsilon\right|^{2}}{|1+2 \varepsilon|^{2}} \cos ^{2}\left(\psi_{i}+\psi,\right) \cos ^{2} \phi$

$\sigma\left(\psi_{i}+\psi_{.} \cdot \bullet\right)=\frac{16 \pi}{9} k^{4}$

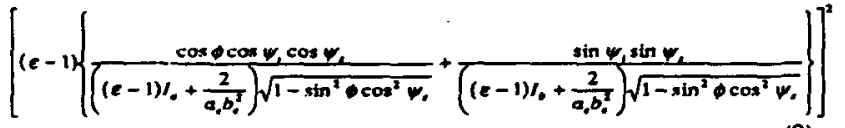

$\sigma^{\prime \prime}\left(\psi_{i}, \psi_{,}, \phi\right)=\frac{4 k h^{2}}{\pi} \cos ^{2} \psi_{i} \sigma\left(\psi_{i}, \phi\right)\left[\frac{\sin \left\{k\left(\sin \psi_{i}+\sin \psi_{j}\right) h\right\}}{k\left(\sin \psi_{i}+\sin \psi_{j}\right) h}\right]^{2}$

where $k$ is the wave number, $a$ is the bubble radius. $\varepsilon$ is the relative permittivity, $h$ is the half length of bubble, $a_{2}$ and $b$, are the semi-axes of the equivalent spheroid, $I_{a}$ and $I_{b}$ are elliptic functions, and $\sigma\left(\psi_{i}, \phi\right)$ is the scattering width of an infinitely long cylinder at oblique incidence.

\section{SIMULATION}

Backscatter from both floating ice and grounded ice have been calculated using this model. For the simulation, the following ice thickness and stratigraphic characteristics were used. The grounded ice consisted of a $10 \mathrm{~cm}$ thick granular layer, a $15 \mathrm{~cm}$ thick clear layer and a $30 \mathrm{~cm}$ thick tubular bubble layer. The floating ice consisted of a $10 \mathrm{~cm}$ thick granular layer, a $60 \mathrm{~cm}$ thick clear layer and a $140 \mathrm{~cm}$ thick tubular bubble layer. In the granular layer the porosity was $1 \%$ and the bubble diameter was $2 \mathrm{~mm}$, while in the tubular layer the porosity was $3 \%$ and the bubbles were $1 \mathrm{~mm}$ in diameter and $5 \mathrm{~cm}$ long. In the simulations the real and imaginary C-band relative dielectric constants for the different materials were as follows: Air 1.0. 0; Ice 3.15, 9.00E-4; Water 65.00, 35.00; Soil 5.00, 0.50.

The results of the simulation are plotted in Fig. 5 which clearly shows the large difference in backscatter intensity between floating and grounded ice. Al the ERS- 1 incidence angle $\left(23^{\circ}\right)$ the backscatter difference is about 7.5 dB. Using ERS-1 SAR data the backscatter difference between floating and grounded ice is -10 to $-12 \mathrm{~dB}$ [Jeffries et al., 1993]. Since the power reflectivity for an ice-water interface is approximately 40 times that of an ice-soil interface, the simulation confirms that backscatter is significantly changed by the presence or absence of a specular ice-water interface.

Backscatter change as a function of ice thickness for an incidence angle of $23^{\circ}$ is shown in Fig.6. In the simulation, the ice had $10 \mathrm{~cm}$ thick granular layer, a $60 \mathrm{~cm}$ thick clear layer and $20-200 \mathrm{~cm}$ thick tubular bubble layer. The porosity and bubble size were the same as those used in the previous simulation. The increase in backscatter with increasing ice thickness. ranging from $-14 \mathrm{~dB}$ to $-6 \mathrm{~dB}$, is similar to that observed by $S A R$ during the period from October 1991 to April 1992 [Jeffries et al., 1993].

Other simulations showed that backscatter increases as bubble radius, bubble length and bubble density increase.

\section{COMPARISON BETWEEN ERS.1 SAR AND MODELLED BACKSCATTER}

The final part of this paper compares simulated and observed backscatter. For the simulation. the ice was divided into sub-layers, and the porosity, mean bubble radius and mean bubble length in each sub-layer as measured in ice cores obtained in April 1992 were used as model input. In order to obtain the observed data. the digital number (DN) for a specific area of a lake was selected from low-resolution data processed at ASF and converted into backscattering coefficients.

The result of the comparison between SAR-derived backscatter and the model output is shown in Fig. 7. At most sites, the model underestimates the backscatter, particularly at sites 7,8 and 11 . The correlation coefficient for a linear regression (Fig. 7) of the two data sets is 0.73 and the model has $-5.5 \mathrm{~dB}$ offset relative to the SAR data.

\section{SUMMARY}

On the basis of ice characteristics obtained from ice core measurements, a numerical model for C-band backscatter from ice growing on shallow tundra lakes has been developed. The model explains the backscatter difference between grounded ice and floating ice, and also the observed increase in backscatter as the ice grows thicker during the winter. Backscattering coefficients derived from ERS-I SAR data were greater than those estimated by the model. The reasons why the model under-estimates backscatter remain to be determined. Further model improvement, such as incorporating a surface roughness model and multi-order bubble scattering, as well as additional field measurement are needed.

\section{ACKNOWLEDGEMENTS}

This work was supported by NASA Polar Program Grant NAG 5-1731 to Jeffries and Weeks. Wakabayashi's participation during his,stay at the Alaska SAR Facility was made possible by NASDA.

\section{REFERENCES}

deLoor, G. P., Dielectric properties of heterogeneous mixtures containing water, J. Microwave Power, 3, 67-73, 1968.

Elachi, C., M. L. Bryan and W. F. Weeks. 1976. Imaging radar observations of frozen arctic lakes, Remote Sens. Environ., S(3). 169-175.

Ruck, G. T., D.E.Barrick, W.D.Stuar, and C.K.Krickbaum, 1970. Radar Cross Section Handbook, vol. 1. New York : Prenum .

Jeffries , M. O. , Wakabayashi, H. . Weeks, W. F., 1993. ERS-I SAR backscatter changes associated with ice growing on shallow lake in arctic Alaska, IGARSS.

Sellmann. P. V.. W. F. Weeks and W. J. Campbell, 1975. Use of sidelooking airborne radar to determine lake depth on the Alaskan North Slope, CRREL Special Report 230.6 pp.. Cold Regions Research and Engineering Laboratory, Hanover, New Hampshire.

Ulaby, F. T. R. K. Moore and A. K. Fung. 1986. Microwave Remote Sensing: Active and Passive, Volume III: Volume Scattering and Emission Theory, Advanced Systems, and Applications. AddisonWesley, Dedham, Massachusetus.

Weeks, W. F., A. G. Fountain, M. L. Bryan and C. Elachi, 1978. Differences in radar return from ice-covered North Slope lakes, $J$. Geophys. Res., 83(C8), 4069-4073.

Weeks, W. F., A. J. Gow and R. J. Schertler, 1981. Ground-truth observations of ice-covered North Slope lakes imaged by radar. CRREL Research Report 81-19,17 pp., Cold Regions Research and Engineering Laboratory, Hanover, New Hampshire.

Weeks, W. F. P. Sellmann and W. J. Campbell, 1977. Interesting features of radar imagery of ice-covered North Slope lakes, $J$. Glaciol., 18(78), 129-136. 


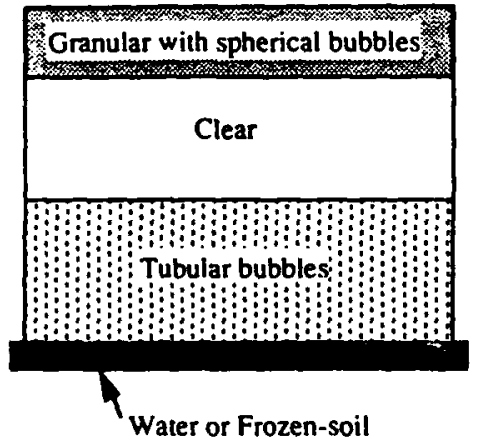

Fig. 1. Typical structure of lake ice as observed in Northern Alaska

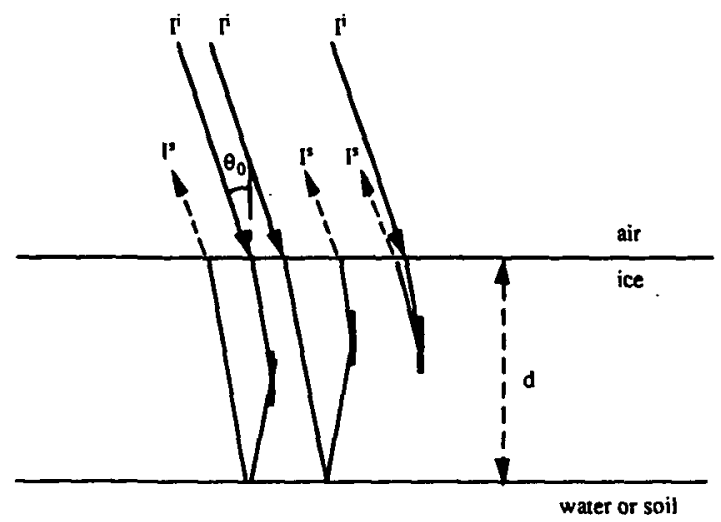

$\theta_{0}$ :incident and scattered angle

d : ice thickness

$I^{i}$ :incident intensity

Is :scantered intensity

Fig. 2. Schematic figure of lake ice reflection including direct backscattering and forward scattering from bubbles.

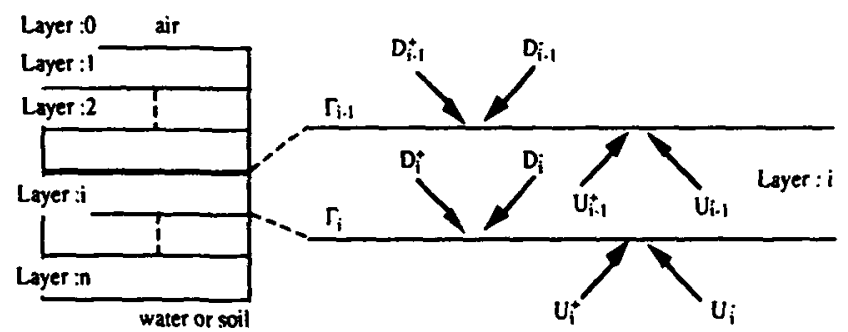

Fig. 3. Multi-layer ice structure

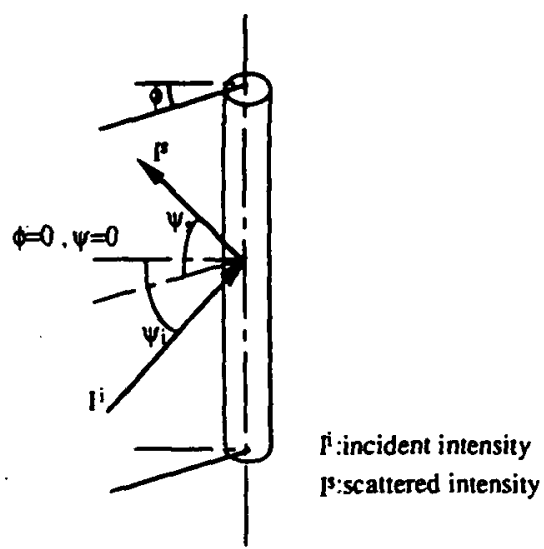

Fig. 4. Bubble geometry

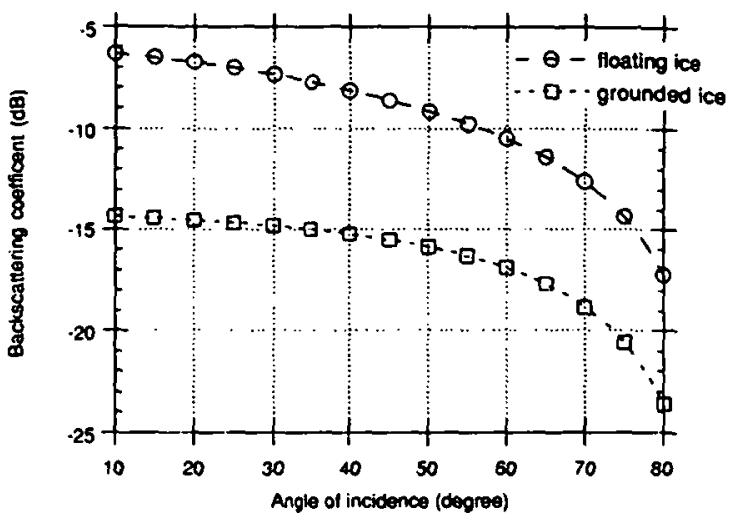

Fig. 5. Backscattering coefficient at $C$-band (VV) versus angle of incidence for the floating ice and the grounded ice.

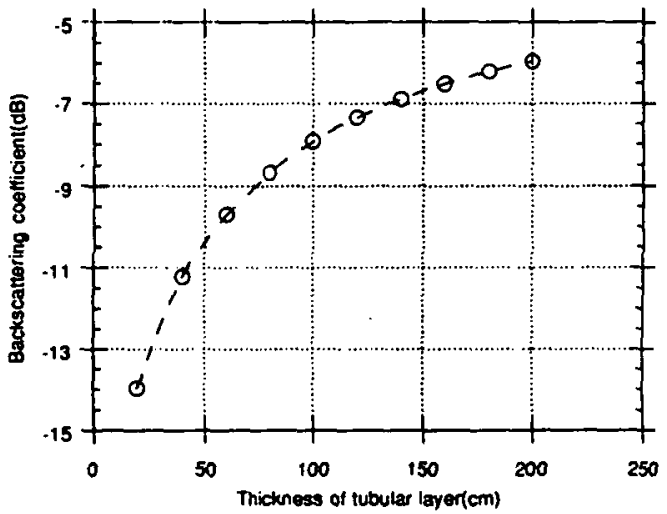

Fig. 6. Backscattering coefficient at C-band (VV) versus the thickness of an ice layer with tubular bubbles.

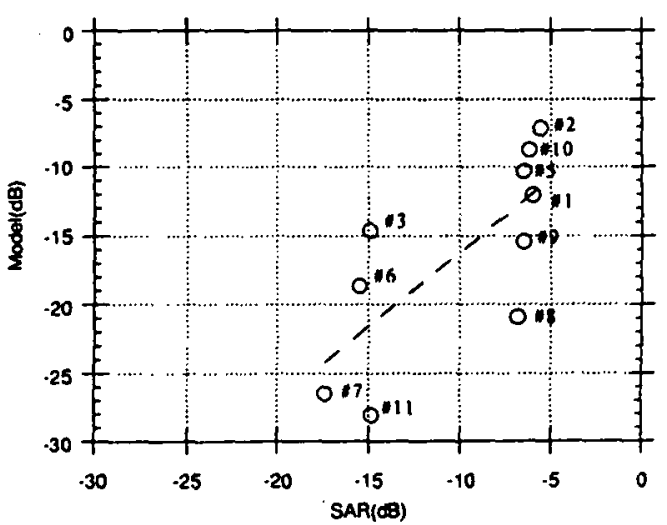

Fig. 7. The backscattering coefficient observed by ERS-I SAR versus the backscattering coefficient estimated by lake ice model. Numbers displayed on the figure indicate the site number. The dotted line is the result of linear curve fitting. 